Discussion paper \#2003-11

Monetary Equilibrium with

Buying and Selling Price Spread without

Transactions Costs

by

Akira Yamazaki

October, 2003 


\title{
Monetary Equilibrium with Buying and Selling Price Spread without Transactions Costs*
}

\author{
Akira YAMAZAKI ${ }^{\dagger}$ \\ Graduate Faculty of Economics \\ Hitotsubashi University
}

October, 2003

\begin{abstract}
This paper is concerned with the Hahn problem in a general monetary equilibrium model at the terminal period. Under the assumption that an initial endowment allocation is not Pareto optimal it is proved that an equilibrium with a positive value of money exists if traders take buying and selling prices of commodities as given even if transactions costs are not explicitly required in the buying and selling activities of traders in commodity markets.

This result seems to suggest two interpretations. One is that a standard monetary equilibrium concept must be strengthened so as to explicitly require an arbitrage-free property of bid-ask spreads of commodity prices vis-à-vis transactions costs. The second interpretation is that a model in which traders take distinct buying and selling prices as given although no transactions costs are required can be thought of as a way to make tax payments required by an external authority in classical papers endogenous in the form of an indirect taxation.
\end{abstract}

*An earlier version of this paper was circulated as "On the Terminal Value of Money without Transactions Costs", RUEE Working Paper \#91-44, Department of Economics, Hitotsubashi University, October, 1991.

$\dagger$ I would like to thank an anonymous referee for his/her useful comments on an earlier draft of this paper. 


\section{Introduction}

This paper is concerned with the Hahn problem in a general monetary equilibrium model at the terminal period ${ }^{1}$ (see Hahn (1965) and Duffie (1990)). Under the assumption that an initial endowments allocation is not Pareto optimal it is proved that an equilibrium with a positive value of money exists if traders take buying and selling prices of commodities as given even if transactions costs are not explicitly required in the buying and selling activities of transactions in commodity markets.

When one introduces pure outside fiat money into a standard general equilibrium model of Arrow-Debreu-McKenzie-Nikaido (ADMN), the fact that an equilibrium exists in the original ADMN model implies that there always exists an equilibrium in which the value of money is zero. But, of course, unless money receives positive valuation in markets, it is impossible to play a basic role as a means of payment. This is the well-known "Hahn problem" in a general monetary equilibrium model pointed out by Hahn (1965). In a series of efforts to solve the problem in 1970's, traders are either simply forced to hold the positive amount of money or motivated to such holdings by required tax payments in the terminal period. (See, e.g., Starr (1974), Kurz (1974a, 1974b), Heller (1974), Okuno (1973), etc.) The reason for such a requirement is that fiat money has no apparent purchasing power in the last period so that traders have no incentives to hold money.

Duffie (1990) in his contribution to this problem has specifically taken up the problem of terminal value of money. In the setting of an ADMN model with outside fiat money and transactions costs expressed by individual transactions possibility sets as in Kurz (1974b) and Heller (1974), he showed that an equilibrium with the positive terminal value of money exists if traders take buying and selling prices in markets as given provided that they have incentives to trade (that is, the initial endowments allocation is not Pareto optimal). Traders are thought to face distinct buying and selling prices due to transactions costs as in a market transactions costs' model of Foley (1970). Duffie's basic idea is that accounting identity forces the total value of purchases to exceed the total value of sales by the amount of outside money if traders are to use the money balances in their transactions at the terminal period.

\footnotetext{
${ }^{1}$ The "Hahn problem" addresses the issue of whether there exists an equilibrium in which the value of money is positive.
} 
An equilibrium is composed of buying prices, selling prices, and preferencemaximizing transactions in traders' budgets given these prices such that the total amount of each commodity (or money) bought does not exceed the total amount of each commodity (or money) sold. This equilibrium concept was introduced by Foley (1970) and Hahn (1971), and others such as Kurz (1974a, 1974b), Starrett (1973), Hayashi (1974), Okuno (1974), Duffie (1990), etc. worked with this definition. In the present paper we employ an exchange economy version of a standard ADMN model as in Duffie (1990) but without explicitly introducing transactions costs in the form of individual transactions possibility sets as is done in his paper as well as in the works of Kurz (1974b) and Heller (1974). The model describes the terminal period of a finite sequence economy. We work with the above equilibrium concept. An existence theorem presented confirms a conjecture of Duffie (1990) that his existence theorem does not seem to rely on the requirement of transactions costs.

Did something go wrong in the setting of transactions costs models? Or, how should we interpret the implication of the result obtained in this paper? There seem to be two possible interpretations. One interpretation is that the equilibrium concept needs to be strengthened so that bid-ask spreads of buying and selling prices do satisfy an arbitrage-free condition defined in an appropriate way vis-à-vis explicitly introduced transactions costs. The second interpretation is to regard bid-ask spreads as representing endogenous indirect taxation. The latter interpretation may be of interest as writers such as Lerner (1947), Starr (1974), Kurz (1974b) and others thought that it is necessary to require that terminal money be used for tax purposes to force traders to demand the terminal money. The formulation of taxes by distinct buying and selling prices without transactions costs do endogenize this taxation.

Finally, let us note that the definition of an equilibrium given above presupposes the free disposability of commodities. By allowing negative buying and selling prices, we do not assume the free disposability. The sign of a bidask spread then depends on the sign of a buying price (see Section 3 on this). We exploit the proof technique of Bergstrom (1976) and Shafer-Sonnenschein (1975) together with the idea of Duffie (1990). 


\section{The Model}

\section{Consumption Characteristics of Agents}

The commodity space is $\mathbb{R}^{\ell}$. For simplicity consumption sets are taken to be $\mathbb{R}_{+}^{\ell}$. Preference relations $\succ \subset \mathbb{R}_{+}^{\ell} \times \mathbb{R}_{+}^{\ell}$ are relatively open, irreflexive, locally nonsatiated, and convex (i.e., the set $\left\{z \in \mathbb{R}_{+}^{\ell} \mid z \succ x\right\}$ is convex for each $x \in \mathbb{R}_{+}^{\ell}$ ).

There are $m$ agents. Each agent $i \in\{1, \ldots, m\}$ is characterized by a preference relation $\succ_{i}$, initial endowments $e_{i} \in \mathbb{R}_{+}^{\ell}$, and an endowment of money $M_{i} \geq 0$.

\section{Market Transactions}

Buying and selling are separate transaction activities which command different price systems. All the prices are expressed in terms of the unit of account. $p^{B} \in \mathbb{R}^{\ell}$ and $p^{S} \in \mathbb{R}^{\ell}$ denote buying and selling prices respectively. Some of the prices may be negative. We express a pair composed of buying prices $p^{B}$ and "reverse-signed" selling prices $-p^{S}$ by $p:=\left(p^{B},-p^{S}\right)$. $p$ represents a vector of prices to be paid for a unit of purchases or sales of commodities in the market. A basic assumption is that each agent takes both buying and selling prices in markets as given and determines its transaction of commodities.

\section{Means of Payment}

Means of payment are fiat money the amount $M=\sum_{i=1}^{m} M_{i}>0$ of which is fixed exogenously. It is implicitly assumed that a public authority is set up for coordinating market transactions and collecting money for its service provided in the markets. The monetary unit is used as the unit of account.

\section{Individual Transaction}

Let $x_{i}^{B}, x_{i}^{S}\left(\in \mathbb{R}_{+}^{\ell}\right)$ represent purchases and sales of $\ell$ commodities by agent $i$ in the markets. Write $x_{i}:=\left(x_{i}^{B}, x_{i}^{S}\right) \in \mathbb{R}_{+}^{\ell} \times \mathbb{R}_{+}^{\ell}$. The transaction of $x_{i}$ by agent $i$ leaves the consumption vector $x_{i}^{C}:=x_{i}^{B}-x_{i}^{S}+e_{i}$ for $i$. It is budget feasible for $i$ at buying and selling prices $p=\left(p^{B},-p^{S}\right) \in \mathbb{R}^{2 \ell}$ if $x_{i}^{C} \geq 0$ and $p \cdot x_{i} \leq M_{i}$. When agent $i$ engages in market transactions $x_{i}$, he receives $p^{S} \cdot x_{i}^{S}$ in money and spends at most $p^{B} \cdot x_{i}^{B}+M_{i}$. Or, one can think of the 
above budget inequality as allowing netting between obligations and claims before the payment of money is made to the market authority.

A budget feasible transaction $x_{i}$ is a preference maximizer for $i$ provided $y_{i}^{C} \nsucc_{i} x_{i}^{C}$ for any budget feasible transaction $y_{i}$ for $i$.

\section{Allocations and Monetary Equilibrium}

A $m$-tuple of transaction vectors $\left(x_{1}, \ldots, x_{m}\right)$ is a transaction allocation if $x_{i} \in \mathbb{R}_{+}^{2 \ell}$ and $x_{i}^{C} \in \mathbb{R}_{+}^{\ell}$ for every $i=1, \ldots, m$. $\left(x_{1}^{C}, \ldots, x_{m}^{C}\right)$ is called the associated consumption allocation. A transaction allocation is feasible if $\sum_{i=1}^{m} x_{i}^{B}=\sum_{i=1}^{m} x_{i}^{S}$. Note that the feasibility of a transaction allocation is equivalent to the feasibility condition $\sum_{i=1}^{m} x_{i}^{C}=\sum_{i=1}^{m} e_{i}$ of the associated consumption allocation $\left(x_{1}^{C}, \ldots, x_{m}^{C}\right)$, which is more familiar.

Given two transaction allocations $\left(x_{1}, \ldots, x_{m}\right)$ and $\left(y_{1}, \ldots, y_{m}\right),\left(x_{1}, \ldots, x_{m}\right)$ is said to Pareto improve $\left(y_{1}, \ldots, y_{m}\right)$ if $x_{i}^{C} \succ_{i} y_{i}^{C}$ for every agent $i$. (This requirement of improvement is stronger than the usual one unless preferences satisfy monotonicity.) A feasible transaction allocation $\left(x_{1}, \ldots, x_{m}\right)$ is Pareto optimal if no other feasible transaction allocations can Pareto improve it.

A monetary equilibrium for the economy $\left(\succ_{i}, e_{i}, M_{i}\right)_{i=1, \ldots, m}$ is a collection $\left(\left(x_{1}, \ldots, x_{m}\right), p\right) \in\left(\mathbb{R}^{2 \ell}\right)^{m} \times \mathbb{R}^{2 \ell}$ such that, given prices $p^{B}, p^{S}$, the transaction $x_{i}$ is budget feasible and a preference maximizer among budget feasible transactions for each $i \in\{1, \ldots, m\}$, and the transaction allocation $\left(x_{1}, \ldots, x_{m}\right)$ is feasible, i.e., $\sum_{i=1}^{m} x_{i}^{B}=\sum_{i=1}^{m} x_{i}^{S}$.

In this definition of a monetary equilibrium buying and selling prices are expressed in terms of the unit of account. Thus, whenever equilibrium prices $p \in \mathbb{R}^{2 \ell}$ exist, the value of money must be positive. One may note that in seeking candidate equilibrium prices of commodities they cannot be constrained to lie in a compact subset of $\mathbb{R}^{2 \ell}$ unless the value of money which might fall to zero is explicitly introduced. It is to be noted that the free disposability of commodities is not assumed so that some of the prices may be negative at equilibrium.

Let us give a statement of a property concerning the preference distribution of an economy which will be needed as a condition of the theorem below.

[Possibility of Individual Utility Enhancement for Feasible Allocations] There is a positive number $k^{*}$ such that for any given feasible consumption allocation $\left(x_{1}^{C}, \ldots, x_{m}^{C}\right), \sum_{i=1}^{m} x_{i}^{C}=\sum_{i=1}^{m} e_{i}$, 
and for any commodity $j,(1 \leq j \leq \ell)$, there is some agent $i \in\{1, \ldots, m\}$ with a consumption vector $0 \leq y \leq k^{*} \sum_{i=1}^{m} e_{i}$ satisfying

(i) $y^{h}=x_{i}^{C h}$ for all $h \neq j$,

(ii) $y \succ_{i} x_{i}^{C}$.

Take any one commodity $j$. If it is distributed freely among agents exhausting all of the resource, then, it should be the case that some one prefers to reduce or increase (within some uniform bound) his/her consumption of the commodity. If there is at least one agent who consumes and regards the commodity $j$ to be desirable (i.e., a "good") or undesirable (i.e., a "bad") in all the range of feasible consumptions, this condition is automatically satisfied. Thus, it is immediate that if all the agents have monotone preferences, the above property is satisfied. Note that it does not rule out some of the commodities to be bads or some of the commodities to become undesirable beyond some levels of their consumption as long as all the agents do not reach their satiation level of a particular commodity simultaneously.

The only case of preference distribution which is ruled out by this condition is the following: There is a commodity $j$ that is regarded desirable only up to some positive consumption levels by all agents, and the sum of the "satiation levels" of the commodity, beyond which an increase of its consumption by agents become undesirable just happens to be exactly equal to the total endowment of the commodity $j$ in the economy. This condition is of a "generic" nature in the sense that even if a particular economy does not satisfy the condition, a slight perturbation of agents' preferences or total endowments will make the condition satisfied.

THEOREM Let $\left(\succ_{i}, e_{i}, M_{i}\right)_{i=1, \ldots, m}$ be an economy with $\sum_{i=1}^{m} e_{i}>0$ and $M=\sum_{i=1}^{m} M_{i}>0$ satisfying the property of the possibility of individual utility enhancement for feasible allocations. Then, there is a monetary equilibrium $\left(\left(x_{1}, \ldots, x_{m}\right), p\right)$ for the economy provided that the transaction allocation $(0, \ldots, 0)$ inducing the initial endowments consumption allocation $\left(e_{1}, \ldots, e_{m}\right)$ is not Pareto optimal.

\section{Discussion and Some Remarks}

A basic scenario supporting the formulation of the model described in the previous section is essentially the same as the one given in Duffie (1990) 
except for the explicit statement of transactions costs in terms of individual transaction possibility sets in case of Duffie.

A market authority is organized and performs the operation of exchanging $\ell$ commodities for money at each point in time for a finite duration. The model describes the terminal period only. The market authority plays the role of a "central banker" and collects money at the time of transactions. Fiat money need not be paper currency but could take the form of "electromoney" in the sense that it is composed of accounts held by traders at the central bank with debits and credits done by electronic devices. Overdrafts are not permitted and we require non-negative balances of money by traders.

Aside from the fact that money being the unit of account, a basic role of money in the model is transactional one at the terminal period. Although it is implicit, money also plays the role of a store of value carried over to the terminal period from the implicit previous time periods in the form of outside money balance.

The theorem asserts that if traders take buying and selling prices of commodities in markets as given, then there is a competitive market equilibrium with a positive monetary value even if no transaction costs are required provided that traders are better off with trades than without trades. This result confirms the conjecture of Duffie that his theorem establishing the existence of monetary equilibrium may obtain without transactions costs (see Duffie (1990, Theorem 1, p.87, and the second paragraph in p.92)).

Let us briefly indicate the nature of buying and selling prices of commodities and the value of money at equilibrium that are given in the proof of the theorem. In our search for equilibrium buying and selling prices and the value of money we normalize buying prices $p^{B}$ to the unit ball $\mathbb{B}^{\ell}$ in $\mathbb{R}^{\ell}$ and express selling prices $p^{S}$ and value of money $p^{M}$ relative to $p^{B}$. The concept of volume of trades is introduced as in Duffie (1990) to determine selling prices. The volume of trades $v$ associated with a transaction vector $x_{i}=\left(x_{i}^{B}, x_{i}^{S}\right) \in \mathbb{R}_{+}^{2 \ell}$ is defined as the vector composed of the maximum number of total purchases or sales of each commodity at markets, that is,

$$
v:=\max \left\{\sum_{i=1}^{m} x_{i}^{B}, \sum_{i=1}^{m} x_{i}^{S}\right\} \in \mathbb{R}_{+}^{\ell}
$$

with the maximum taken coordinatewise. At equilibrium we have $\sum_{i=1}^{m} x_{i}^{B}=$ $\sum_{i=1}^{m} x_{i}^{S}=v$.

Given volume of trade $v \in \mathbb{R}_{+}^{\ell}$, selling prices $p^{S}$ associated with buying 
prices $p^{B}$ are defined via bid-ask spread factor $\delta^{j}\left(p^{B}, v\right)$ given by

$$
\delta^{j}\left(p^{B}, v\right):=\frac{\operatorname{sgn}\left(p^{B j}\right) v^{j}}{1+v^{j}}
$$

for $j=1, \ldots, \ell$. The bid-ask spread of commodity $j$ is then $\delta^{j}\left(p^{B}, v\right) p^{B j}$. In other words, the selling price $p^{S j}$ of commodity $j$ is given by $\left(1-\delta^{j}\left(p^{B}, v\right)\right) p^{B j}$. The absolute value of bid-ask spread factor of a commodity is strictly less than one, and it is monotonic in volumes of trades of that commodity. One might feel that the latter property is somewhat counter to what one might expect as a property of bid-ask spreads. Note, however, that the spirit of bidask spreads in the present model is that they originate in transactions costs which certainly accumulate as volumes of trades increase. Bid-ask spreads are designed so that a positive buying price commands a positive spread and a negative price a negative spread. This means that for desired commodities buyers pay more than sellers receive, and for undesired commodities, in order to have them disposed, sellers pay more than buyers receive.

During the course of the existence proof in Section 4, given buying prices $p^{B}$, selling prices $p^{S}$ are determined according to bid-ask spreads given in the previous paragraph. The value $p^{M}$ of money is set so that the value of existing stock of outside money is exactly equal to the total sum of the bid-ask spreads resulting from the volume $v$ of trades in markets. It means that agents use outside money to finance the excess of their payments over receipts in order to clear the results of their transactions with the market authority in commodity markets.

By the hypothesis of the theorem the initial endowments allocation is not Pareto optimal. Thus, agents do wish to engage in trades provided bid-ask spreads are sufficiently small. Bid-ask spreads introduced above have the property that they can be made arbitrarily small in neighborhoods of the initial endowments allocation. It therefore follows that there will be a positive volume of trades at equilibria. By the way value of money is determined in our model, a positive volume of trades induces a positive terminal value of money.

Nevertheless, the theorem may at first seem counterintuitive. Since the model deals with the terminal period, it must be transaction demands that give rise to the positivity of monetary value. Market transactions of commodities must require costs if they are to motivate transactions demands for money. Thus, a natural question might arise: Why is it that one can establish the existence of monetary equilibrium without requiring transactions costs? 
The answer is straightforward. It is because we assumed that agents take buying and selling prices as given no matter how big bid-ask spreads are. But agents will in general take advantage of bid-ask spreads unless their arbitrage transactions are unprofitable due to existing transactions costs. Therefore, the fact that the theorem is true seems to point to the need for strengthening the equilibrium concept adopted by us and others in the context of a general equilibrium model with transactions costs. It is implicitly assumed in the literature ( see, e.g., Foley (1970) and Duffie (1990, p.90) ) that transactions costs are severe enough to prevent arbitrage over bid-ask spreads. What need to be done thus seems to be to require explicitly bid-ask spreads of prices be arbitrage free in the sense that arbitrage transactions are not profitable visà-vis transactions costs incurred by such activity. It would lead to rethinking of the formulation of the model with individual transactions technologies.

We would like to note that writers such as Lerner (1947), Hahn (1971), Starr (1974), Heller (1974), and others have either simply forced agents to demand fiat money at the terminal period or motivated such holdings requiring the payment of exogenously given lump sum taxes. In this context we could regard bid-ask spreads of prices in the present model as endogenous indirect taxes by the authority. Spread factor $\delta^{j}$ then represents a quantity tax levied on commodity $j$. One is thus led to feel that it is natural to have existence of a monetary equilibrium in a framework without explicit transactions costs. And a conceptual difficulty pointed out in the previous paragraph would not arise in this interpretation.

A final remark may be due. The model in this paper does not suggest why fiat money may offer transactional advantages as a medium of exchange, but given a positive value for money the advantages are not difficult to imagine, considering for instance the work of Kiyotaki and Wright (1989), and Ostroy and Starr (1973). Instead the model presented here merely suggests why outside fiat money may have positive value in the first place even in a situation where explicit transactions costs do not exist. 


\section{Proof of the Theorem}

1. Let $k^{*}$ be the positive number given in the statement of the property of diversified satiation of consumption, and let

$$
\bar{e}:=\max \left\{k^{*}, 2\right\} \sum_{i=1}^{m} e_{i}>0 .
$$

For each $i=1, \ldots, m$, define

$$
X_{i}:=[0, \bar{e}] \times[0, \bar{e}] \subset \mathbb{R}_{+}^{\ell} \times \mathbb{R}_{+}^{\ell}
$$

where $[0, \bar{e}]:=\left\{z \in \mathbb{R}^{\ell} \mid 0 \leq z^{j} \leq \bar{e}^{j}\right.$ for all $\left.j=1, \ldots, \ell\right\}$. Let us also define

$$
X:=\prod_{i=1}^{m} X_{i} \text { and } V:=[0, \bar{e}] \subset \mathbb{R}_{+}^{\ell} .
$$

$\mathrm{X}$ is the space of transaction allocations and an element $v \in V$ will be called volume of trades which will be defined later.

$\mathbb{B}^{\ell}$ denotes the unit closed ball in $\mathbb{R}^{\ell}$ centered at the origin 0 , and a vector $q$ in $\mathbb{B}^{\ell}$ will represent buying prices of $\ell$ commodities. Given buying prices $q$ and volume of trades $v$, selling prices are defined via a spread factor $\delta^{j}(q, v)$, $j=1, \ldots, \ell$, between purchases and sales prices. Let

$$
\delta^{j}(q, v):=\frac{\operatorname{sgn}\left(q^{j}\right) v^{j}}{1+v^{j}}
$$

for each $j=1, \ldots, \ell$. Define a diagonal matrix $\Lambda(q, v)$ as a $\ell \times \ell$ matrix with diagonal elements $1-\delta^{j}(q, v), j=1, \ldots, \ell$. Then, the selling price vector associated with $q$ is set to be given by $\Lambda(q, v) q$. Thus, spreads between buying and selling prices $q-\Lambda(q, v) q$ is equal to $\left(\delta^{1}(q, v) q^{1}, \ldots, \delta^{\ell}(q, v) q^{\ell}\right)$. The spread factor $\delta^{j}(q, v)$ has the following property:

(i) buying price is greater or less than selling price depending upon whether it is positive or negative;

(ii) non-zero buying and selling prices are identical if and only if there are no trades in the markets, i.e., $v^{j}=0$;

(iii) $\left|\delta^{j}(q, v)\right|$ is increasing in $v^{j}$ and $0 \leq\left|\delta^{j}(q, v)\right|<1$. 
It follows from (1) that for each $j=1, \ldots, \ell$ we have

$$
\begin{aligned}
\delta^{j}(q, v) q^{j} & \geq 0 ; \\
\delta^{j}(q, v) q^{j} v^{j} & >0 \text { if and only if } q^{j} v^{j} \neq 0 .
\end{aligned}
$$

Given a vector $q \in \mathbb{B}^{\ell}$ of buying prices, the following notation will be used to denote the vector of buying prices and reverse-signed selling prices:

$$
q(v):=(q,-\Lambda(q, v) q) \in \mathbb{R}^{\ell} \times \mathbb{R}^{\ell}
$$

Now define

$$
\begin{aligned}
b^{M} & :=\frac{m}{M} \sum_{j=1}^{\ell} \bar{e}^{j}, \\
\Delta^{M} & :=\left[0, b^{M}\right] \subset \mathbb{R}_{+} .
\end{aligned}
$$

$p^{M} \in \Delta^{M}$ will be the value of money. $b^{M}$ is an upper bound for $p^{M}$. In our proof here buying prices are "normalized" to the unit closed ball, and selling prices and the value of money are expressed relative to buying prices.

2. We now define correspondences $\beta_{i}, \varphi_{i}, \mu_{i}$. Let $\left(x, v, q, p^{M}\right) \in X \times$ $V \times \mathbb{B}^{\ell} \times \Delta^{M}$. For $i=1, \ldots, m$

$$
\begin{aligned}
& \beta_{i}\left(x, v, q, p^{M}\right):=\left\{z_{i} \in X_{i} \mid q(v) \cdot z_{i} \leq p^{M} M_{i}+1-\|q\|, z_{i}^{C} \geq 0\right\} . \\
& \beta_{m+1}\left(x, v, q, p^{M}\right):=\mathbb{B}^{\ell} .
\end{aligned}
$$

For $i=1, \ldots, m$

$$
\begin{aligned}
\varphi_{i}\left(x, v, q, p^{M}\right):=\left\{z_{i} \in X_{i} \mid z_{i}^{C} \succ_{i} x_{i}^{C}\right\}, \\
\varphi_{m+1}\left(x, v, q, p^{M}\right):=\left\{\bar{q} \in \mathbb{B}^{\ell} \mid \bar{q}(v) \cdot\left[\sum_{i=1}^{m} x_{i}-(v,-v)\right],\right. \\
\left.\quad>q(v) \cdot\left[\sum_{i=1}^{m} x_{i}-(v, v)\right]\right\}, \\
\mu_{m+2}\left(x, v, q, p^{M}\right):=\max \left\{\sum_{i=1}^{m} x_{i}^{B}, \sum_{i=1}^{m} x_{i}^{S}\right\},
\end{aligned}
$$$$
\text { where the maximum is taken coordinatewise, }
$$$$
\mu_{m+3}\left(x, v, q, p^{M}\right):=\left\{\frac{q(v) \cdot(v, v)}{M}\right\} .
$$

$\beta_{i}$ and $\varphi_{i}, i=1, \ldots, m$, are budget and preference correspondences constrained to $X_{i}=[0, \bar{e}]$. The correspondence $\mu_{m+2}$ defines the volume of 
trades. $\mu_{m+3}$ sets the value of money so that the total value of money balance is exactly equal to the amount of money needed to pay for the difference between buying and selling values of commodities in carrying out total trades. $\varphi_{m+1}$ sets the buying prices (and hence the selling prices) of commodities in such a way that the value of total excess demand for money in the economy is maximized. (Let us recall that $\bar{q}(v)$ in the definition of $\varphi_{m+1}$ is defined as in the equation (4) with $q$ replaced by $\bar{q}$. Keep in mind that this convention is used again when we write $q^{*}\left(v^{*}\right)$ and $q\left(v^{*}\right)$ in (10) that are defined as in (4) with $q$ and/or $v$ replaced by $q^{*}$ and/or $v^{*}$.)

3. A slightly modified version of an abstract equilibrium existence theorem due to Shafer-Sonnenschein (1975) will be applied to the correspondences introduced above.

Given non-empty finite sets $N_{1}$ and $N_{2}$, a pair $\left\{\left(Z_{i}, \varphi_{i}, \beta_{i}\right)_{i \in N_{1}},\left(Z_{i}, \mu_{i}\right)_{i \in N_{2}}\right\}$ composed of a family of ordered triples and a family of ordered pairs, where $\varphi_{i}: Z \rightarrow Z_{i}, \beta_{i}: Z \rightarrow Z_{i}$, and $\mu_{i}: Z \rightarrow Z_{i}$ are correspondences with $Z=\prod_{i \in N_{1} \cup N_{2}} Z_{i}$, is called a bisectoral social system. An equilibrium for the bisectoral social system is an $x \in Z$ satisfying $x_{i} \in \beta_{i}(x), \varphi_{i}(x) \cap \beta_{i}(x)=\emptyset$ for all $i \in N_{1}$, and $x_{i} \in \mu_{i}(x)$ for all $i \in N_{2}$.

Equilibrium Existence Lemma: If, for every $i \in N_{1} \cup N_{2}$, the set $Z_{i}$ is a non-empty, compact, convex subset of a Euclidean space, $\varphi_{i}$ is an open graph correspondence from $Z$ to $Z_{i}$ such that for every $z \in Z, z_{i} \notin \operatorname{co} \varphi_{i}(z)$, and $\beta_{i}$ and $\mu_{i}$ are non-empty and convex-valued correspondences from $Z$ to $Z_{i}$ where $\beta_{i}$ 's are continuous and $\mu_{i}$ 's are upper hemi-continuous, then the bisectoral social system $\left\{\left(Z_{i}, \varphi_{i}, \beta_{i}\right)_{i \in N_{1}},\left(Z_{i}, \mu_{i}\right)_{i \in N_{2}}\right\}$ has an equilibrium.

The lemma follows from the proof of a theorem in Shafer-Sonnenschein (1975). We shall show later that $\beta_{i}, \varphi_{i}, \mu_{i}$ correspondences introduced in the previous step satisfy all the conditions of the above lemma. Thus, by applying the equilibrium existence lemma to the bisectoral social system defined by $\beta_{i}, \varphi_{i}(i=1, \ldots, m+1)$ and $\mu_{i}(i=m+2, m+3)$, one obtains 


$$
\begin{aligned}
& \xi^{*}=\left(x^{*}, v^{*}, q^{*}, p^{* M}\right) \in X \times V \times \mathbb{B}^{\ell} \times \Delta^{M} \text { satisfying } \\
& q^{*} \in \mathbb{B}^{\ell} \\
& q^{*}\left(v^{*}\right) \cdot x_{i}^{*} \leq p^{* M} M_{i}+1-\left\|q^{*}\right\| \\
& x_{i}^{* C} \geq 0 \quad \text { for } \quad i=1, \ldots, m \\
& q^{*}\left(v^{*}\right) \cdot\left[\sum_{i=1}^{m} x_{i}^{*}-\left(v^{*}, v^{*}\right)\right] \geq q\left(v^{*}\right) \cdot\left[\sum_{i=1}^{m} x_{i}^{*}-\left(v^{*}, v^{*}\right)\right] \\
& \text { for all } q \in \mathbb{B}^{\ell} \\
& \varphi_{i}\left(\xi^{*}\right) \cap \beta_{i}\left(\xi^{*}\right)=\emptyset \quad \text { for } i=1, \ldots, m+1 \\
& v^{*}=\max \left\{\sum_{i=1}^{m} x_{i}^{* B}, \sum_{i=1}^{m} x_{i}^{* S}\right\} \\
& p^{* M}=\frac{q^{*}\left(v^{*}\right) \cdot\left(v^{*}, v^{*}\right)}{M}
\end{aligned}
$$

4. Let us proceed to show the feasibility of the transaction allocation $x^{*}=\left(x_{1}^{*}, \ldots, x_{m}^{*}\right)$ and the nonzero property of commodity (buying) prices $q^{*}$.

We first show

$$
\sum_{i=1}^{m} x_{i}^{* B}=\sum_{i=1}^{m} x_{i}^{* S}=v^{*} .
$$

If $\sum_{i} x_{i}^{* B} \neq \sum_{i} x_{i}^{* S}$, then we must have $\left(\sum_{i} x_{i}^{* B}-v^{*}\right) \neq 0$ or $\left(\sum_{i} x_{i}^{* S}-v^{*}\right) \neq 0$. It therefore follows from (10) that

$$
q^{*}\left(v^{*}\right) \cdot\left[\sum_{i=1}^{m} x_{i}^{*}-\left(v^{*}, v^{*}\right)\right]>0
$$

and $\left\|q^{*}\right\|=1$. Hence, one obtains from (8) and (13) that

$$
q^{*}\left(v^{*}\right) \cdot\left[\sum_{i=1}^{m} x_{i}^{*}-\left(v^{*}, v^{*}\right)\right] \leq q^{*}\left(v^{*}\right) \cdot \sum_{i=1}^{m} x_{i}^{*}-p^{* M} M \leq 0
$$

which contradicts the above strict inequality. This establishes (14).

We now show that in (8) one actually has

$$
q^{*}\left(v^{*}\right) \cdot x_{i}^{*}=p^{* M} M_{i}+1-\left\|q^{*}\right\| \quad \text { for } i=1, \ldots, m .
$$

Recall that (14) implies

$$
\sum_{i=1}^{m} x_{i}^{* C}=\sum_{i=1}^{m} e_{i}
$$

which in turn implies

$$
0 \leq x_{i}^{* C} \leq \sum_{i=1}^{m} e_{i}<\bar{e} \text { for any } i=1, \ldots, m .
$$


Thus, if we had $q^{*}\left(v^{*}\right) \cdot x_{i}^{*}<p^{* M} M_{i}+1-\left\|q^{*}\right\|$ for some $i$, then by the local nonsatiation of preferences we would obtain $\varphi_{i}\left(\xi^{*}\right) \cap \beta_{i}\left(\xi^{*}\right) \neq \emptyset$, which contradicts (11).

It now follows from (13), (14), and (15) that

$$
\left\|q^{*}\right\|=1 \text {. }
$$

In addition to (18) one can prove

$$
q^{* j} \neq 0 \quad \text { for every } \quad j=1, \ldots, \ell .
$$

Indeed, assume we had $q^{* h}=0$ for some $1 \leq h \leq \ell$. It follows from (17) and the property of the possibility of individual utility enhancement for feasible allocations that for some agent $i$ there is a consumption vector $y \in \mathbb{R}_{+}^{\ell}$ such that $y^{j}=x_{i}^{* C j}$ for all $j \neq h$ and $y \succ_{i} x_{i}^{* C}$. Put $z^{B}=\left(y-e_{i}\right)^{+}$and $z^{S}=\left(y-e_{i}\right)^{-}$where for any $t \in \mathbb{R}^{\ell}, t^{+}:=\max \{t, 0\}$ and $t^{-}:=\max \{-t, 0\}$ with the maximum taken coordinatewise. Write $z=\left(z^{B}, z^{S}\right)$. Then, we have $z^{C}=z^{B}-z^{S}+e_{i}=y \geq 0, z^{B j}-z^{S j}=x_{i}^{* B j}-x_{i}^{* S j}$ for all $j \neq h$, $z^{B}=\left(z^{B}-z^{S}\right)^{+}$, and $z^{S}=\left(z^{B}-z^{S}\right)^{-}$. To check $z$ is budget feasible for $i$, define $J_{B}:=\left\{j \mid x_{i}^{* B j}>x_{i}^{* S j}\right\}$ and $J_{S}:=\left\{j \mid x_{i}^{* B j}<x_{i}^{* S j}\right\}$. Then, since $q^{* h}=0$ we have

$$
\begin{aligned}
q^{*}\left(v^{*}\right) \cdot & x_{i}^{*}-q^{*}\left(v^{*}\right) \cdot z \\
= & \sum_{j=1}^{\ell}\left(q^{* j} x_{i}^{* B j}-\left(1-\delta^{j}\left(q^{*}, v^{*}\right)\right) q^{* j} x_{i}^{* S j}\right)-\sum_{j \in J_{B}} q^{* j} \\
& \times\left(x_{i}^{* B j}-x_{i}^{* S j}\right)+\sum_{j \in J_{S}}\left(1-\delta^{j}\left(q^{*}, v^{*}\right)\right) q^{* j}\left(x_{i}^{* S j}-x_{i}^{* B j}\right) \\
= & \sum_{j \in J_{B}} \delta^{j}\left(q^{*}, v^{*}\right) q^{* j} x_{i}^{* S j}+\sum_{j \in J_{S}} \delta^{j}\left(q^{*}, v^{*}\right) q^{* j} x_{i}^{* B j} \geq 0
\end{aligned}
$$

where the last inequality follows from the property of $\delta^{j}$ described by (2). This shows $z$ is budget feasible for $i$. Hence, $z^{C} \succ_{i} x_{i}^{* C}$ contradicts (11). Thus (19) must be true.

5. We now show that

$$
p^{* M}>0 .
$$

If $p^{* M}=0,(13)$ implies

$$
q^{*}\left(v^{*}\right) \cdot\left(v^{*}, v^{*}\right)=\sum_{j=1}^{\ell} \delta^{j}\left(q^{*}, v^{*}\right) q^{* j} v^{* j}=0 .
$$


It then follows from (2), (3), and (19) that $v^{*}=0$ which implies by (12)

$$
\begin{aligned}
x_{i}^{* B}=x_{i}^{* S} & =0 \text { for all } i=1, \ldots, m, \text { and } \\
q^{*}\left(v^{*}\right) & =\left(q^{*},-q^{*}\right) .
\end{aligned}
$$

Since the transaction allocation $(0,0)$ is not Pareto optimal by the hypothesis of the theorem, there exists a feasible transaction allocation $\left(x_{1}, \ldots, x_{m}\right)$ that Pareto improves $\left(x_{1}^{*}, \ldots, x_{m}^{*}\right)$ so that $x_{i}^{C} \succ_{i} x_{i}^{* C}$ for all $i$ and $\sum_{i=1}^{m} x_{i}^{B}=$ $\sum_{i=1}^{m} x_{i}^{S}$. But (11) implies that for all $i=1, \ldots, m$ we must have

$$
q^{*} \cdot x_{i}^{B}-q^{*} \cdot x_{i}^{S}=q^{*}\left(v^{*}\right) \cdot x_{i}>p^{* M} M_{i} \geq 0 .
$$

One thus obtains $q^{*} \cdot\left(\sum_{i=1}^{m} x_{i}^{B}-\sum_{i=1}^{m}-x_{i}^{S}\right)>0$ contradicting the feasibility of the transaction allocation $\left(x_{1}, \ldots, x_{m}\right)$. Therefore, we must have (20). This argument also establishes

$$
v^{*} \neq 0
$$

6. Let $\bar{e}_{k}:=k \bar{e}$ for each positive integer $k=1,2, \ldots$ and replace $\bar{e}$ by $\bar{e}_{k}$ in Steps $1-5$. Then, the arguments in the previous steps establish the existence of $\xi_{k}^{*}=\left(x_{k}^{*}, v_{k}^{*}, q_{k}^{*}, p_{k}^{* M}\right)$ satisfying the properties $(7)-(21)$ where $\xi^{*}$ is replaced by $\xi_{k}^{*}$. We show $\xi_{k}^{*}, k=1,2, \ldots$, are bounded. For this purpose we show that for every $i=1, \ldots, m$ one has

$$
\left(x_{i}^{* B j}\right)\left(x_{i}^{* S j}\right)=0 \text { for all } j=1, \ldots, \ell
$$

where the suffix $k$ is omitted from now on in our present step of arguments. Put $y_{i}^{B}:=\left(x_{i}^{* B}-x_{i}^{* S}\right)^{+}, y_{i}^{S}:=\left(x_{i}^{* B}-x_{i}^{* S}\right)^{-}$, and $y_{i}:=\left(y_{i}^{B}, y_{i}^{S}\right)$. Then, we have $0 \leq y_{i} \leq x_{i}^{*}$ and $y_{i}^{C}=x_{i}^{* C}$. Define sets, $J_{B}$ and $J_{S}$, of indices of commodities as in Step 4. We prove the property (22) by showing $y_{i}=x_{i}^{*}$. Assume $y_{i} \neq x_{i}^{*}$. Then, we must have $x_{i}^{* S j}>0$ for some $j \in J_{B}$ or $x_{i}^{* B j}>0$ for some $j \in J_{S}$. It therefore follows from (2), (3), (19), and (21) that

$$
\begin{aligned}
q^{*} & \left(v^{*}\right) \cdot x_{i}^{*}-q^{*}\left(v^{*}\right) \cdot y_{i} \\
& =\sum_{j=1}^{\ell}\left(q^{* j}\left(v^{*}\right) x_{i}^{* B j}-\left(1-\delta^{j}\left(q^{*}, v^{*}\right)\right) q^{* j}\left(v^{*}\right) x_{i}^{* S j}\right) \\
& -\sum_{j \in J_{B}} q^{* j}\left(v^{*}\right)\left(x_{i}^{* B j}-x_{i}^{* S j}\right)+\sum_{j \in J_{S}}\left(1-\delta^{j}\left(q^{*}, v^{*}\right)\right) q^{* j}\left(v^{*}\right)\left(x_{i}^{* B j}-x_{i}^{* S j}\right) \\
& \geq \sum_{j \in J_{B}} \delta^{j}\left(q^{*}, v^{*}\right) q^{* j}\left(v^{*}\right) x_{i}^{* S j}+\sum_{j \in J_{S}} \delta^{j}\left(q^{*}, v^{*}\right) q^{* j}\left(v^{*}\right) x_{i}^{* B j} \\
& >0 .
\end{aligned}
$$


Thus, one has $q^{*}\left(v^{*}\right) \cdot y_{i}<p^{* M} M_{i}$. But since $y_{i}^{C}=x_{i}^{* C}$, by (17) it contradicts (11) and the local nonsatiation of preferences. This establishes (22).

Now, for every $i=1, \ldots, m$, one has $x_{i}^{* B}-x_{i}^{* S}+e_{i}=x_{i}^{* C} \geq 0$. Thus, for each $i$

$$
x_{i}^{* S}-x_{i}^{* B} \leq e_{i} \leq \sum_{i=1}^{m} e_{i} .
$$

In view of (22), (23) implies that

$$
0 \leq x_{i}^{* S} \leq e_{i} \leq \sum_{i=1}^{m} e_{i} \text { for every } i=1, \ldots, m .
$$

By (14), (24) implies

$$
\begin{aligned}
0 \leq x_{i}^{* B} \leq \sum_{i=1}^{m} x_{i}^{* B}= & \sum_{i=1}^{m} x_{i}^{* S} \leq \sum_{i=1}^{m} e_{i} \\
& \text { for every } i=1, \ldots, m .
\end{aligned}
$$

Since (24) and (25) hold for all $k=1,2, \ldots$, it follows from (13) and (14) that $\xi_{k}^{*}=\left(x_{k}^{*}, v_{k}^{*}, q_{k}^{*}, p_{k}^{* M}\right), k=1,2, \ldots$, are bounded. Let us denote a limit point of the sequence $\xi_{k}^{*}$ by $\xi^{*}=\left(x^{*}, v^{*}, q^{*}, p^{* M}\right)$ again and assume without loss of generality that the sequence $\xi_{k}^{*}$ itself converges to $\xi^{*}$. As every $\xi_{k}^{*}$ satisfies (13), (14), (15) and (18), so does $\xi^{*}$. The argument in Step 4 to obtain (19) also applies to the limit point $\xi^{*}$, and hence so does the argument in Step 5 to obtain (20).

Replacing $X_{i}$ by $X_{i}^{*}:=\mathbb{R}_{+}^{\ell} \times \mathbb{R}_{+}^{\ell}$, define $\beta_{i}^{*}$ and $\varphi_{i}^{*}$ for each $i=1, \ldots, m$ just as $\beta_{i}$ and $\varphi_{i}$ are defined in Step 2. Then, one must have

$$
\varphi_{i}^{*}\left(\xi^{*}\right) \cap \beta_{i}^{*}\left(\xi^{*}\right)=\emptyset \quad \text { for } \quad i=1, \ldots, m,
$$

because, otherwise, one would have

$$
\varphi_{i}\left(\xi_{k}^{*}\right) \cap \beta_{i}\left(\xi_{k}^{*}\right) \neq \emptyset
$$

for $k$ large enough in contradiction to (11) since each $x_{i}$ on the budget hyperplane has local cheaper points as to be shown in the last step of our proof.

7. Define $p^{*}=\left(p^{* B},-p^{* S}\right)$ by

$$
\begin{aligned}
p^{* B j} & =\frac{q^{* j}}{p^{* M}} \quad \text { for } \quad j=1, \ldots, \ell \text { and } \\
p^{* S j} & =\frac{\left(1-\delta^{j}\left(q^{*}, v^{*}\right)\right) q^{* j}}{p^{* M}} \quad \text { for } \quad j=1, \ldots, \ell .
\end{aligned}
$$


Then, by $(14),(15),(18),(20)$, and $(26),\left(\left(x_{1}^{*}, \ldots, x_{m}^{*}\right), p^{*}\right)$ is a monetary equilibrium. It remains to show that the correspondences $\beta_{i}, \varphi_{i}, \mu_{i}$ introduced in Step 2 satisfy the conditions of the Equilibrium Existence Lemma.

8. The nonempty-valuedness of $\beta_{m+1}$ and $\mu_{i}, i=m+2, m+3$, is trivial, and that of $\beta_{i}, i=1, \ldots, m$, follows from $p^{M} \geq 0, q \in \beta^{\ell}$, and $0 \in X_{i}$. Note that in the lemma $\varphi_{i}$ 's are allowed to be empty-valued. The upper hemi-continuity of $\mu_{i}, i=m+2, m+3$, and the continuity of $\beta_{m+1}$ are immediate. That $\varphi_{i}$ has an open graph is straightforward for $i=m+1$ and is a consequence of the continuity of preferences for $i=1, \ldots, m$. The upper hemi-continuity of $\beta_{i}, i=1, \ldots, m$, being straightforward, the only property one needs to show is the lower hemi-continuity of $\beta_{i}, i=1, \ldots, m$. For this purpose, it is sufficient to prove existence of local cheaper points for any transaction vector lying on a budget hyperplane. We state it as a lemma.

Lemma: $\quad$ Given $x_{i} \in \mathbb{R}_{+}^{\ell} \times \mathbb{R}_{+}^{\ell}, q \in \mathbb{B}^{\ell}, p^{M} \geq 0, v \in \mathbb{R}_{+}^{\ell} \times \mathbb{R}_{+}^{\ell}$ satisfying $q(v) \cdot x_{i}=p^{M} M_{i}+1-\|q\|$, there is a sequence $\left\{x_{i n}\right\}_{n}$ in $X_{i}$ such that $x_{i n} \rightarrow x_{i}$ and $q(v) \cdot x_{i n}<q(v) \cdot x_{i}$ for all $n$.

The lemma is an immediate consequence of the following fact:

Fact: Let $p(\neq 0) \in \mathbb{R}^{n}$ and $H(p)=\left\{z \in \mathbb{R}^{n} \mid p \cdot z=0\right\}$. If one has $H(p) \cap \mathbb{R}_{++}^{n}=\emptyset$, then $p \in \mathbb{R}_{+}^{n} \cup \mathbb{R}_{-}^{n}$.

Let $p \neq 0$ and $H(p) \cap \mathbb{R}_{++}^{n}=\emptyset$. Let $J_{+}=\left\{j \mid p^{j}>0\right\}, J_{0}=\left\{j \mid p^{j}=0\right\}$, and $J_{-}=\left\{j \mid p^{j}<0\right\}$. If the conclusion of the lemma were false, then one would have $p \notin \mathbb{R}_{+}^{n} \cup \mathbb{R}_{-}^{n}$. It would imply $J_{+} \neq \emptyset$ and $J_{-} \neq \emptyset$. Let $x \in \mathbb{R}^{n}$ be defined by

$$
x^{j}= \begin{cases}1 & \text { for } \quad j \in J_{0} \\ \frac{1}{p^{j}} & \text { for } \quad j \in J_{+} \\ -\frac{\# J_{+}}{\left(\# J_{-}\right) p^{j}} & \text { for } \quad j \in J_{-}\end{cases}
$$

where \# indicates the number of elements in a set. Then, $x \in \mathbb{R}_{++}^{n}$ and $p \cdot x=0$, i.e., $x \in H(p)$, contradicting $H(p) \cap \mathbb{R}_{++}^{n}=\emptyset$. This proves the above fact.

Let $x_{i}, q, p^{M}$, and $v$ be given as in the lemma. Since $q(v)=(q,-\Lambda(q, v) q) \notin$ $\mathbb{R}_{+}^{2 \ell} \cup \mathbb{R}_{-}^{2 \ell}$, by applying the above fact to the hyperplane $H(q(v)) \subset \mathbb{R}^{2 \ell}$, one 
obtains $y_{i} \in \mathbb{R}_{++}^{2 \ell}$ such that $q(v) \cdot y_{i}<0$. By shortening the length of the vector $y_{i}$ if necessary, one can assume w.1.o.g. $y_{i} \in X_{i}$. Now, for each $i=1,2, \ldots$ define $x_{i n}:=x_{i}+\frac{1}{n}\left(y_{i}-x_{i}\right) \in X_{i}$. Then, one has $q(v) \cdot x_{i n}<p^{M} M_{i}+1-\|q\|$ and $x_{i n} \rightarrow x_{i}$.

This establishes the lemma and completes the proof of the theorem. 


\section{References}

[1] T.C. BERGSTROM, "How to discard 'Free Disposability' - at No Cost," Journal of Mathematical Economics, 3 (1976), 131-134.

[2] D. DUFFIE, "Money in General Equilibrium Theory," in B.M. Friedman and F.H. Kahn eds.: Handbook of Monetary Economics, Vol. I, Elsevier Science Publishers, 1990, 81-100.

[3] D. FOLEY, "Economic Equilibrium with Costly Marketing," Journal of Economic Theory, 2 (1970), 276-291.

[4] F. HAHN, "On Some Problems of Proving the Existence of an Equilibrium in a Monetary Economy," in F. Hahn and F. Brechling eds.: The Theory of Interest Rates, Macmillan, London, 1965, 126-135.

[5] F. HAHN, "Equilibrium with Transaction Costs," Econometrica, 39 (1971), 417-439.

[6] T. HAYASHI, "The Non-Pareto Efficiency of Initial Allocation of Commodities and Monetary Equilibrium: An Inside Money Economy," Journal of Economic Theory, 7 (1974), 173-187.

[7] W. HELLER, "The Holding of Money Balances in General Equilibrium," Journal of Economic Theory, 7 (1974), 93-108.

[8] M. KURZ, "Arrow-Debreu Equilibrium of an Exchange Economy with Transaction Cost," International Economic Review, 15 (1974), 699-717.

[9] N. KIYOTAKI AND R. WRIGHT, "On Money as a Medium of Exchange," Journal of Political Economy, 97 (1989), 927-954.

[10] M. KURZ, "Equilibrium with Transaction Cost and Money in a Single Market Exchange Economy," Journal of Economic Theory, 7 (1974b), 418452 .

[11] A. LERNER, "Money as a Creature of the State," Proceedings of American Economic Association, 37 (1947), 312-317.

[12] M. OKUNO, "Essays on Monetary Equilibrium in a Sequence of Markets," Technical Report 120, Institute for Mathematical Studies in the Social Sciences, Stanford University, 1973. 
[13] J. OSTROY AND R. STARR, "Money and the Decentralization of Exchange," Econometrica, 42 (1973), 1093-1113.

[14] W. SHAFER AND H. SONNENSCHEIN, "Equilibrium in Abstract Economies without Ordered Preferences," Journal of Mathematical Economics, 2 (1975), 345-348.

[15] R. STARR, "The Structure of Exchange in Barter and Monetary Economies," Quarterly Journal of of Economics, 86 (1972), 290-302.

[16] R. STARR, "The Price of Money in a Pure Exchange Monetary Economy with Taxation," Econometrica, 42 (1974), 45-54.

[17] D. STARRETT, "Inefficiency and the Demand for 'Money' in a Sequence Economy," Review of Economic Studies, 40 (1973), 437-448.

[18] A. YAMAZAKI, "Monetary Equilibria in a Continuum Economy with General Transaction Technologies," RUEE Working Paper 89-39, Hitotsubashi University, 1989.

[19] A. YAMAZAKI, "Equilibrium in Economies with Incomplete Markets and Outside Money: Transactions Costs and Existence," Department of Economics, Hitotsubashi University, 1991. 